\title{
SPEN induces miR-4652-3p to target HIPK2 in nasopharyngeal carcinoma
}

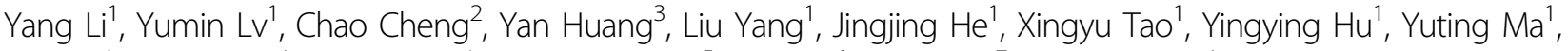 \\ Yun Su', Liyang Wu', Guifang Yư ${ }^{4}$, Qingping Jiang ${ }^{5}$, Shu Liư ${ }^{6}$ Xiong Liu ${ }^{7}$ and Zhen Liu
}

\begin{abstract}
SPEN family transcriptional repressor (SPEN), also known as the SMART/HDAC1-associated repressor protein (SHARP), has been reported to modulate the malignant phenotypes of breast cancer, colon cancer, and ovarian cancer. However, its role and the detail molecular basis in nasopharyngeal carcinoma (NPC) remain elusive. In this study, the SPEN mRNA and protein expression was found to be increased in NPC cells and tissues compared with nonmalignant nasopharyngeal epithelial cells and tissues. Elevated SPEN protein expression was found to promote the pathogenesis of NPC and lead to poor prognosis. Knockdown of SPEN expression resulted in inactivation ofPI3K/AKT and c-JUN signaling, thereby suppressing NPC migration and invasion. In addition, miR-4652-3p was found to be a downstream inducer of SPEN by targeting the homeodomain interacting protein kinase 2 (HIPK2) gene, a potential tumor suppressor that reduces the activation of epithelial-mesenchymal transition (EMT) signaling, thereby reducing its expression and leading to increased NPC migration, invasion, and metastasis. In addition, SPEN was found to induce miR-4652-3p expression by activating PI3K/AKT/C-JUN signaling to target HIPK2. Our data provided a new molecular mechanism for SPEN as a metastasis promoter through activation of PI3K/AKT signaling, thereby stimulating the c-JUN/miR-4652-3p axis to target HIPK2 in NPC.
\end{abstract}

\section{Introduction}

Nasopharyngeal carcinoma (NPC) is highly prevalent in Southern China with a much higher incidence than elsewhere ${ }^{1}$. It is characterized by high invasion and early metastasis. Patients with NPC are often diagnosed at advanced stage of the disease ${ }^{2}$. Although regional control has been greatly improved by the advances in radiotherapy and chemotherapy, metastasis remains the major

\footnotetext{
Correspondence: Xiong Liu (liux1218@126.com) or

Zhen Liu (narcissus_jane@163.com)

${ }^{1}$ Affiliated Cancer Hospital \& Institute of Guangzhou Medical University, Guangzhou Municipal and Guangdong Provincial Key Laboratory of Protein Modification and Degradation, State Key Laboratory of Respiratory Disease, School of Basic Medical Sciences, Guangzhou Medical University, Guangzhou 510095 Guangdong, China

${ }^{2}$ Department of Pediatric Otorhinolaryngology, Shenzhen Key Laboratory of Viral Oncology, The Clinical Innovation \& Research Centre, Shenzhen Hospital, Southern Medical University, Shenzhen, Guangdong, China

Full list of author information is available at the end of the article

These authors contributed equally: Yang Li, Yumin Lv, Chao Cheng, Yan Huang Edited by G. Calin
}

cause of treatment failure ${ }^{3}$. Accordingly, understanding the molecular mechanisms by of invasion and/or metastasis of NPC is critical for the identification of novel therapeutic targets and formulation of better treatment strategies.

In previous studies, several genes have been reported to be involved in NPC metastasis ${ }^{4-6}$. SPEN family transcriptional repressor (SPEN), also known as SMART/ HDAC1-associatedrepressor (SHARP), is a large nuclear protein that plays an important role in transcriptional regulation and inactivation of chromosome $\mathrm{X}^{7}$. Legare et al. reported that the inactivation of SPEN may contribute to breast tumor progression and thus suggested SPEN as a tumor suppressor in ER $\alpha$-positive breast cancers $^{8}$. In contrast, Feng et al. found that SPEN (SHARP) gene acts as a candidate oncogene, promoting the pathogenesis of human hematopoietic malignancies, breast and colon cancer' ${ }^{9}$. Furthermore, Liu et al. demonstrated that SPOCD1(SPEN) may act as a 
carcinogenesis factor by activating the PI3K/AKT pathway to restrained cell apoptosis in Ovarian cancer $(\mathrm{OC})^{10}$. These studies suggested that SPEN played a significant and complexed role in tumor pathogenesis. However, the molecular alterations and biological functional involvement of SPEN in the pathogenesis of NPC have not been investigated.

MicroRNAs (miRNAs) area class of small (17-23 nucleotides) noncoding RNAs that silence mRNA molecules through a degradation or translational inhibition process. They participate in various biological processes, including tumorigenesis and metastasis ${ }^{11-13}$. Multiple miRNAs have been found to play key roles in regulating the expression of various critical genes during the development of human tumors ${ }^{4,14,15}$. Several of them were identified as regulators of the progression of NPC, such as miR-374a, miR-184, and miR-3188 $8^{6,16,17}$. However, the regulation of miRNAs involving SPEN has not been reported to date.

This study reports a newly discovered miRNA, namely miR-4652-3p, as an oncogenic regulator miRNA, which was found to be upregulated by the potential oncogene
SPEN through the activation of PI3K/AKT/c-JUN signaling. In addition, miR-4652-3p was found to directly target HIPK2 to participate in the SPEN-mediated promotion of NPC migration, invasion, and metastasis.

\section{Results}

SPEN expression and clinicopathological characteristics in NPC

To determine the role of SPEN in NPC development, its expression level was analyzed in various NPC cell lines (HONE1, SUNE1, 5-8F, 6-10B, CNE1, and CNE2) and immortalized nasopharyngeal epithelial (NP) cell lines (NP69 and SXSW-1489) by quantitative real-time polymerase chain reaction (qRT-PCR) analysis. The endogenous mRNA level of SPEN in all six NPC cell lines was significantly upregulated compared with that in SXSW1489 nonmalignant immortalized NP cells, although the difference between NPC cells and NP69 nonmalignant NP cells (Fig. 1a) was not significant. As for protein level, a large cohort of 238 NPC tissues and 54 nonmalignant NP tissues were examined by immunohistochemistry (IHC) analysis. SPEN expression displayed nuclear and
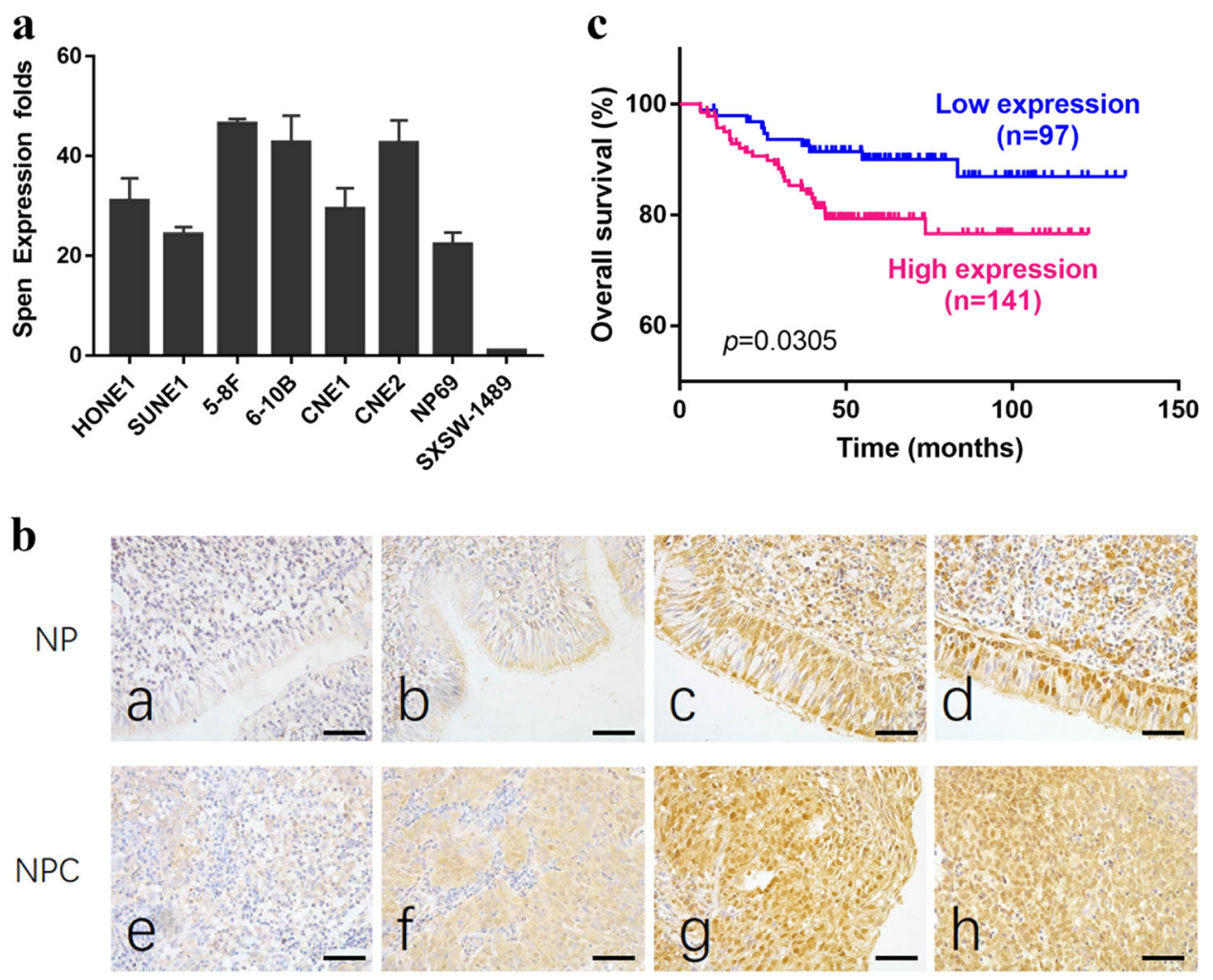

Fig. 1 Elevated expression of SPEN promoted the poor prognosis of NPC patients. a mRNA levels of SPEN analyzed by qRT-PCR assays in six human NPC cell lines (HONE1, SUNE1, 5-8F, 6-10B, CNE1, CNE2) and immortalized normal nasopharyngeal epithelial cell lines NP69 and SXSW-1489. b Representative IHC images of SPEN expression in NP and NPC tissues. a, b: weak expression of SPEN in NP samples; $c$, d: strong and positive expression of SPEN in NP samples; e, f: weak staining of SPEN in NPC specimens; $g$, h: strong and positive staining of SPEN in NPC specimens. (original magnification $\times 400)$. c Kaplan-Meier survival curve for overall survival in NPC patients based on SPEN expression level $(P=0.0305$, log-rank test). 
Table 1 The expression of SPEN in NPC compared with NP tissues.

\begin{tabular}{llllll}
\hline Group & Cases $(\boldsymbol{n})$ & \multicolumn{2}{l}{ SPEN expression } & $\boldsymbol{X}^{2}$ & $\boldsymbol{P}^{*}$ value \\
\cline { 3 - 5 } & & $\begin{array}{l}\text { High } \\
\text { expression }\end{array}$ & $\begin{array}{l}\text { Low } \\
\text { expression }\end{array}$ & & \\
\hline NPC & 238 & $141(59.2 \%)$ & $97(40.8 \%)$ & 29.234 & 0.000 \\
NP & 54 & $10(18.5 \%)$ & $44(81.5 \%)$ & & \\
\hline
\end{tabular}

NPC nasopharyngeal carcinoma, NP nasopharyngeal epithelium.

${ }^{*} X^{2}$ test was applied to assess the expression of SPEN in NPC and NP

cytoplasmic distribution patterns in both NPC and NP cells with different expression levels (Fig. 1b). Statistical analysis confirmed that among the 238 NPC specimens, 97 (40.8\%) had low SPEN expression and 141 (59.2\%) had high SPEN expression. Instead, among the 54 NP tissues, low SPEN-expressing tissues accounted for 44 (81.5\%), and high SPEN-expressing tissues accounted for 10 (18.5\%). In addition, NPC tissues showed higher SPEN expression level than NP tissues $(P<0.001$, Table 1$)$. In addition, the analysis of the relationship between SPEN expression and the clinicopathological characteristics in patients with NPC revealed no statistically significant association between SPEN expression level and patient age and gender, although SPEN expression was positively correlated with the $\mathrm{N}$ (lymph node metastasis) stage $(P<0.001 ; \mathrm{N} 0-\mathrm{N} 1$ vs. N2-N3), $T$ (tumor size) stage $(P=0.021 ; \mathrm{T} 1-\mathrm{T} 2$ vs. T3-T4) and clinical stage $(P<0.001$; I-II vs. III-IV, Table 2). Survival analysis revealed that low-SPEN expressing patients had longer overall survival than high SPEN-expressing patients. $(P=0.0305$, Fig. 1c).

\section{Knockeddown expression of SPEN suppresses cell migration and invasion in vitro and inactivates PI3K/AKT and C-JUN signaling}

RNA interference was conducted to knock down SPEN expression in HONE1 and 5-8F cells. SPEN gene expression analysis by qRT-PCR confirmed that, after silencing, its expression was significantly decreased in NPC cells compared with their control cells (Fig. 2a). After SPEN knockdown, the expression of p-PI3K and $\mathrm{p}$-AKT was largely abrogated, as well as the expression of c-JUN (Fig. 2b). In addition, Transwell, Boyden and wound-healing assays to investigate the effect of silencing SPEN on the migration and invasion abilities, NPC cells showed that downregulation of SPEN in HONE1 and 5-8F NPC cells markedly inhibited cell migration and invasion abilities (Fig. 2c, d). Taken together, these findings revealed that inhibition of SPEN decreased NPC cell migration and invasion as well as inactivated PI3K/AKT and c-JUN signaling.

\section{SPEN induces miR-4652-3p expression in NPC cells}

To investigate the downstream effector miRNAs regulated by SPEN, an Affymetrix 3.0 miRNA array was used to examine the differential expression of miRNAs between HONE1-siSPEN and HONE1-NC cells (Fig. 3a). Expression analysis by qRT-PCR confirmed that miR4652-3p expression was downregulated by twofold or more at the mRNA level in SPEN-silenced HONE1 cells compared with HONE1-NC group (Fig. 3b).

\section{MiR-4652-3p promotes NPC cell metastasis in vitro and in vivo}

HONE1 and 5-8F cells successfully transfected with miR-4652-3pmimics were used to investigate the effects of miR-465-3p on migration and invasion alterations in vitro. First, qRT-PCR analysis showed the expression level of miR-4652-3p was higher in HONE1 and 5-8F cells transfected with miR-4652-3p mimics than that in cells transfected with NC (Fig. 3c). In addition, overexpression of miR-4652-3p in HONE1 and 5-8F cells greatly promoted cell migration and invasion abilities (Fig. $3 \mathrm{~d}, \mathrm{e})$. In addition, we established two NPC cell lines stably overexpressing miR-4652-3p, namely HONE1-LVmiR-4652-3p and 5-8F-LV-miR-4652-3p, by lentivirusmediated transfection and used them for in vivo studies. Pulmonary metastasis were performed in animal models, developed by injecting NPC cell lines stably overexpressing miR-4652-3p via the tail vein of nude mice. Metastatic nodules in the lung of nude mice were detected under a fluorescence microscope and confirmed by histological analysis. More pulmonary metastases were detected in the miR-4652-3p overexpressing group than in the control (NC) group. No metastases were detected in heart, liver, spleen, kidney, brain, and other organs (Fig. 3f).

\section{Suppression of miR-4652-3p expression inhibits NPC cell migration and invasion}

The biological role of miR-4652-3p in NPC pathogenesis was further investigated by introducing miR-4652-3p inhibitors into the HONE1-LV-miR-4652-3p and 5-8FLV-miR-4652-3p cells stably overexpressing miR-4652$3 p$. The expression level of miR-4652-3p was found to be elevated in the NPCLV-miR-4652-3p cells compared with LV-NC group, while miR-4652-3p inhibitors significantly reduced the miR-4652-3p expression level (Fig. 4a). Consistent with the role of miR-4652-3p in migration and invasion, inhibition of miR-4652-3p expression by its specific inhibitor suppressed cell migration and invasion in miR-4652-3p-overexpressing HONE1 and 5-8F cells (Fig. 4c, d). The analysis of the mechanism revealed that miR-4652-3p overexpression upregulated the protein expression levels of $\mathrm{N}$-cadherin and vimentin, but downregulated E-cadherin protein level. On the other 
Table 2 Correlation between the clinicopathologic characteristics and expression of SPEN in NPC.

\begin{tabular}{|c|c|c|c|c|c|}
\hline \multirow[t]{2}{*}{ Characteristics } & \multirow[t]{2}{*}{ Cases $(n)$} & \multicolumn{2}{|l|}{ SPEN expression } & \multirow[t]{2}{*}{$x^{2}$} & \multirow[t]{2}{*}{$P^{*}$ value } \\
\hline & & High expression & Low expression & & \\
\hline \multicolumn{6}{|l|}{ Gender } \\
\hline Male & 181 & 108 (59.7\%) & $73(40.3 \%)$ & 0.056 & 0.812 \\
\hline Female & 57 & $33(57.9 \%)$ & $24(42.1 \%)$ & & \\
\hline \multicolumn{6}{|l|}{ Age (years) } \\
\hline$\leq 50$ & 153 & $92(60.1 \%)$ & 61 (39.9\%) & 0.140 & 0.709 \\
\hline$>50$ & 85 & $49(57.6 \%)$ & $36(42.4 \%)$ & & \\
\hline \multicolumn{6}{|l|}{ N classification } \\
\hline No-N1 & 122 & 47 (38.5\%) & $75(61.5 \%)$ & 44.502 & 0.000 \\
\hline N2-N3 & 116 & $94(81.0 \%)$ & $22(19.0 \%)$ & & \\
\hline \multicolumn{6}{|l|}{ T classification } \\
\hline $\mathrm{T} 1-\mathrm{T} 2$ & 110 & $63(57.2 \%)$ & 47 (42.8\%) & 5.331 & 0.021 \\
\hline T3-T4 & 108 & $78(72.2 \%)$ & $30(27.8 \%)$ & & \\
\hline \multicolumn{6}{|l|}{ Clinical stage } \\
\hline$|-| \mid$ & 64 & $25(39.0 \%)$ & $39(61.0 \%)$ & 14.766 & 0.000 \\
\hline III-IV & 174 & $116(66.7 \%)$ & 58 (33.3\%) & & \\
\hline
\end{tabular}

NPC Nasopharyngeal carcinoma, NP normal epithelium.

${ }^{*} X^{2}$ test was applied to access the associations between SPEN expression and the clinicopathological parameters.

hand, inhibition of miR-4652-3p expression led to opposite effect on the expression levels of these epithelial-mesenchymal transition (EMT) related proteins (Fig. 4b).

\section{MiR-4652-3p directly targets HIPK2}

To further explore the mechanisms by which miR-46523 p promotes NPC cell migration and invasion, various miRNA target prediction software were used to predict miR-4652-3p target genes, including TargetScan, miRWalk, and mirDIP. A total of 262 potential targets were found in the above-mentioned three databases, which include HIPK2 (Fig. 5a). Western blot analysis confirmed that the HIPK2 protein level was downregulated by miR4652-3p overexpression, and upregulated by miR-4652-3p inhibitors (Fig. 5b). Simultaneously, co-transfection of miR-4652-3pmimics and HIPK2 $3^{\prime}$ untranslated region (UTR) WT sequence significantly decreased the luciferase reporter activity, whereas the miR-4652-3p inhibitor had the opposite effect. These effects on luciferase activity were abrogated when cells were co-transfected with mutated HIPK2 3'UTR (Fig. 5c). Furthermore, depletion of HIPK2 induced the expression of vimentin, but reduced E-cadherin expression, indicating that HIPK2 silencing restored the EMT signaling suppression caused by inhibition of miR-4652-3p (Fig. 5d). Moreover, HIPK2 expression level was increased after the silencing SPEN in
NPC cells, indicating that HIPK2 is involved in the SPEN/ miR-4652-3p-induced NPC cell metastasis (Fig. 5e).

\section{SPEN induces miR-4652-3p expression by modulating $\mathrm{PI3K} / \mathrm{AKT} / \mathrm{c}-J U N$ signaling}

To determine the transcriptional regulatory mechanisms of miR-4652-3p expression, the University of California Santa Cruz and Profiler of Multi-Omic data online software were used to analyze a $2-\mathrm{kb}$ upstream region to the transcription start site of miR-4652-3p. A potential c-JUN binding site was predicted at -696 to -708 (Fig. 6a). Accordingly, c-JUN plasmids were introduced into NPC cells, and its effect on miR-4652-3p expression was evaluated by qRT-PCR analysis and Western blot analysis (Fig. $6 b, c)$. The results of the qRT-PCR analysis indicated that miR-4652-3p expression was greatly elevated in HONE1 and $5-8$ F cells transfected with $\mathrm{c}-J \mathrm{UN}$ plasmids, suggesting that c-JUN acts as an upstream regulator of miR-4652-3p (Fig. 6d). The binding of c-JUN to the miR-4652-3p promoter region was further confirmed by chromatin immunoprecipitation (ChIP) analysis (Fig. 6e). In a subsequent experiment, LY294002, a specific inhibitor of PI3K, was used to block the PI3K expression in HONE1 and $5-8$ F cells and its effect on miR-4652-3p function was evaluated. Western blot and qRT-PCR analyses demonstrated that LY294002 decreased the levels of p-PI3K, p-AKT, c-JUN, and miR-4652-3p, but increased HIPK2 


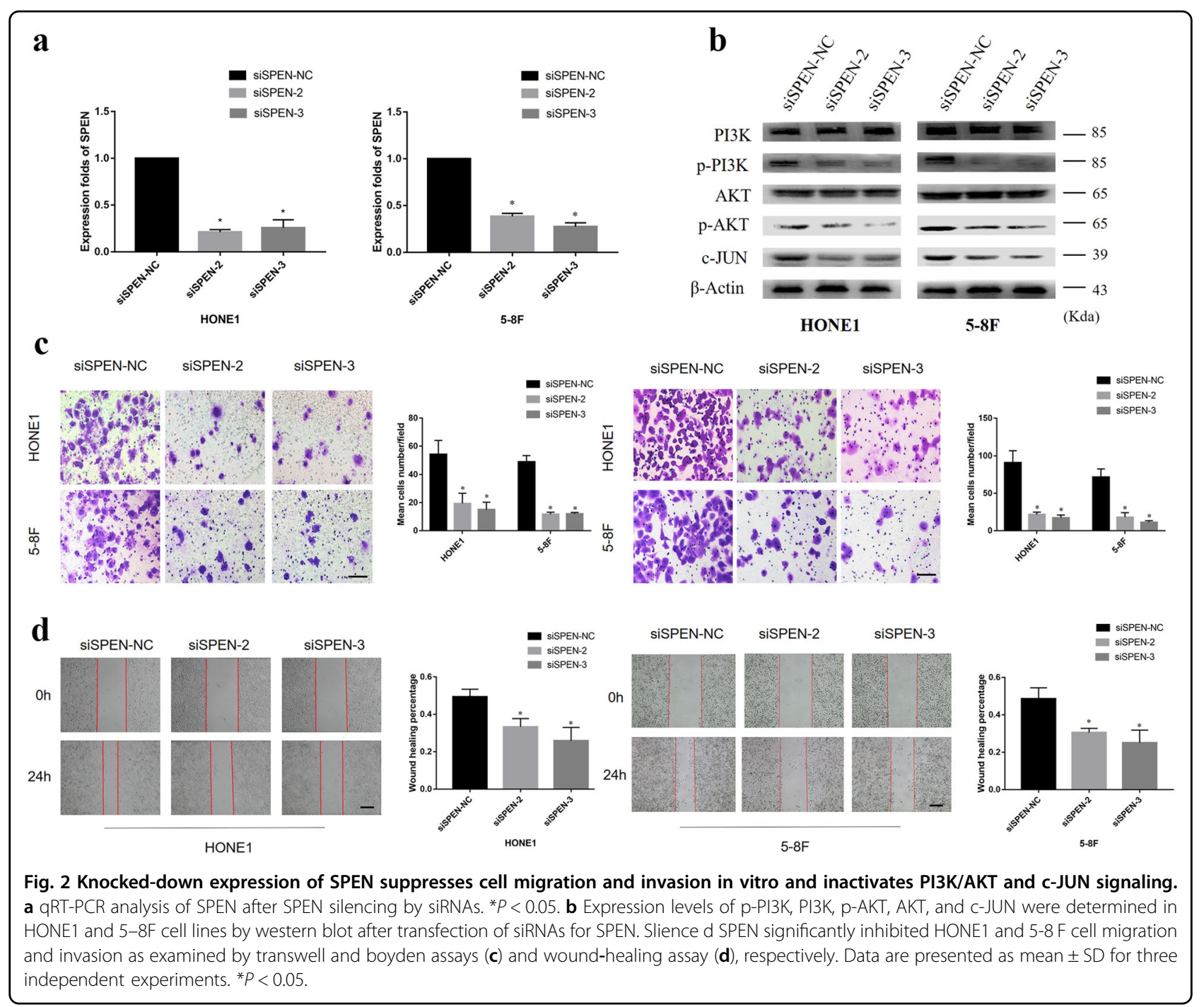

expression compared with the control groups (Fig. 6f, g). Our results showed that SPEN induced miR-4652-3p expression by activating PI3K/AKT/c-JUN signaling.

\section{Materials and methods}

\section{Cell lines and cell cultures}

The six NPC cell lines (5-8F, 6-10B, CNE1, CNE2, HONE1, and SUNE1) and immortalized nonmalignant human nasopharyngeal epithelial NP69 cells used in this study were obtained from the Cancer Research Institute of Southern Medical University (Guangzhou, China). The immortalized nonmalignant human nasopharyngeal epithelial cell line SXSW-1489 was purchased from SHBIO (SiXin, Shanghai, China). In this study, the NPC and SXSW1489 cells were cultured in RPMI-1640 medium supplemented with $10 \%$ fetal bovine serum (FBS; HyClone, Logan, UT, USA). NP69 cells were cultured in a defined keratinocyte serum-free medium supplemented with epidermal growth factor (EGF, Invitrogen, Carlsbad, CA, USA).

\section{RNA isolation and qRT-PCR}

Total RNA from each sample was quantified by the NanoDrop2000 and converted to cDNA. cDNA was added to RT 2 Profiler $^{\mathrm{TM}}$ PCR Array (TAKARA, JAPAN) and ran in Real-Time PCR instrument (Applied Biosystems, Thermo Fisher Scientific, NY, USA). Primer sequence SPEN (Forward: CGAGCATTTCAAACGATATGGC; Reverse: CCATTTT GTTGACCGAGTTGTG), c-JUN (Forward: GTGCCGAA AAAGGAAGCTGG, Reverse: CTGCGTTAGCATGAGTT GGC), HIPK2 (Forward: CGGACTGGAGAAATACGCA, Reverse: ACAGATGACTGGTGCTGCTTAC), miR-46523p (GCGGTTCTGTTAACCCATCCCCTCA) were synthesized and purchased by Sangon Biotech (Shanghai, China).

\section{Western blot analysis}

Whole cell lysates of NPC cells were prepared with a proteinase and phosphatase inhibitor cocktail (Roche, CA, USA). Equal amounts of proteins were resolved to $10 \%$ SDS-polyacrylamide gel electrophoresis and transferred to 


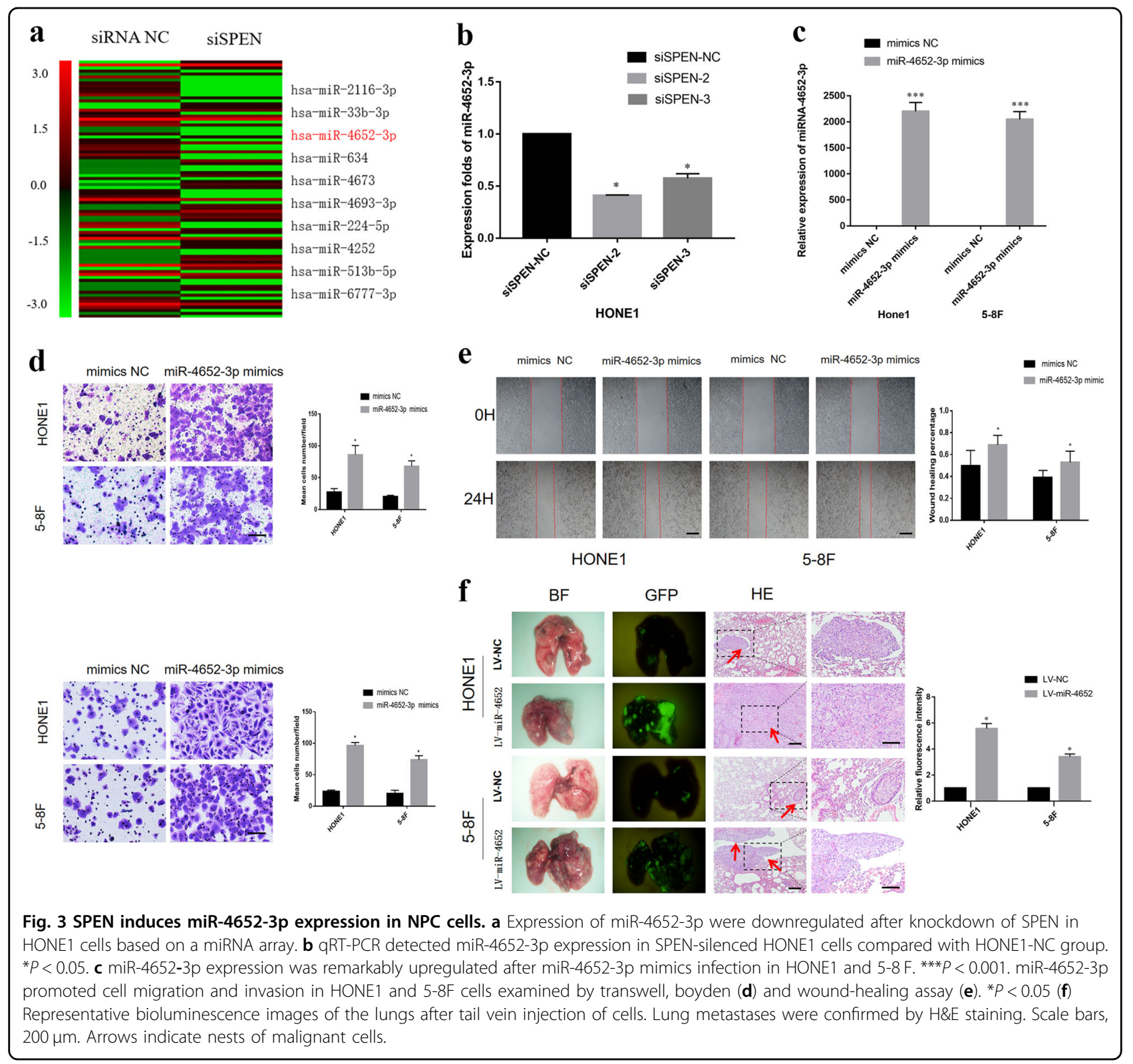

PVDF membranes (Millipore, Danvers, MA, USA) and blocked with $5 \%$ nonfat dry milk in Tris-buffered saline, $\mathrm{pH}$ 7.5. Membranes were immuno-blotted overnight at $4{ }^{\circ} \mathrm{C}$. Protein blots were probed with primary antibodies against HIPK2 (Abcam \#ab108543), E-cadherin (Cell Signaling Technology \#3195), N-cadherin (Cell Signaling Technology \#13116), SPEN (Abcam \#ab72266), c-JUN (Abcam \#ab40766), phosphor-AKT (Cell Signaling Technology \#4060), AKT (Cell Signaling Technology \#4691), phosphor-PI3K (Cell Signaling Technology \#17366), and PI3K (Cell Signaling Technology \#4292). Goat anti-rabbit IgG HRP-linked antibody $(1: 10,000)$ and goat anti-mouse IgG HRP-linkedantibody $(1: 10,000)$ were from Proteintech (Rosemont, IL, USA). Signals were visualized by chemiluminescence (Bio-rad, Hercules, California) and quantitated using a Quantity One system (Bio-Rad, Hercules, CA, USA).

\section{Immunohistochemical staining}

Two hundred and thirty-eight (238) paraffin-embedded NPC specimens and fifty-four (54) cases of nonneoplastic nasopharyngeal mucosal tissue were collected from Nanfang Hospital of Southern Medical University without any therapy before sampling. Among 238 NPC cases, 181 cases are male and 57 are female, ranged from 20 to 84 years old of age (median 58.9 years old). The clinical process was approved by the Ethics Committees of Nanfang Hospital of Southern Medical University and the 


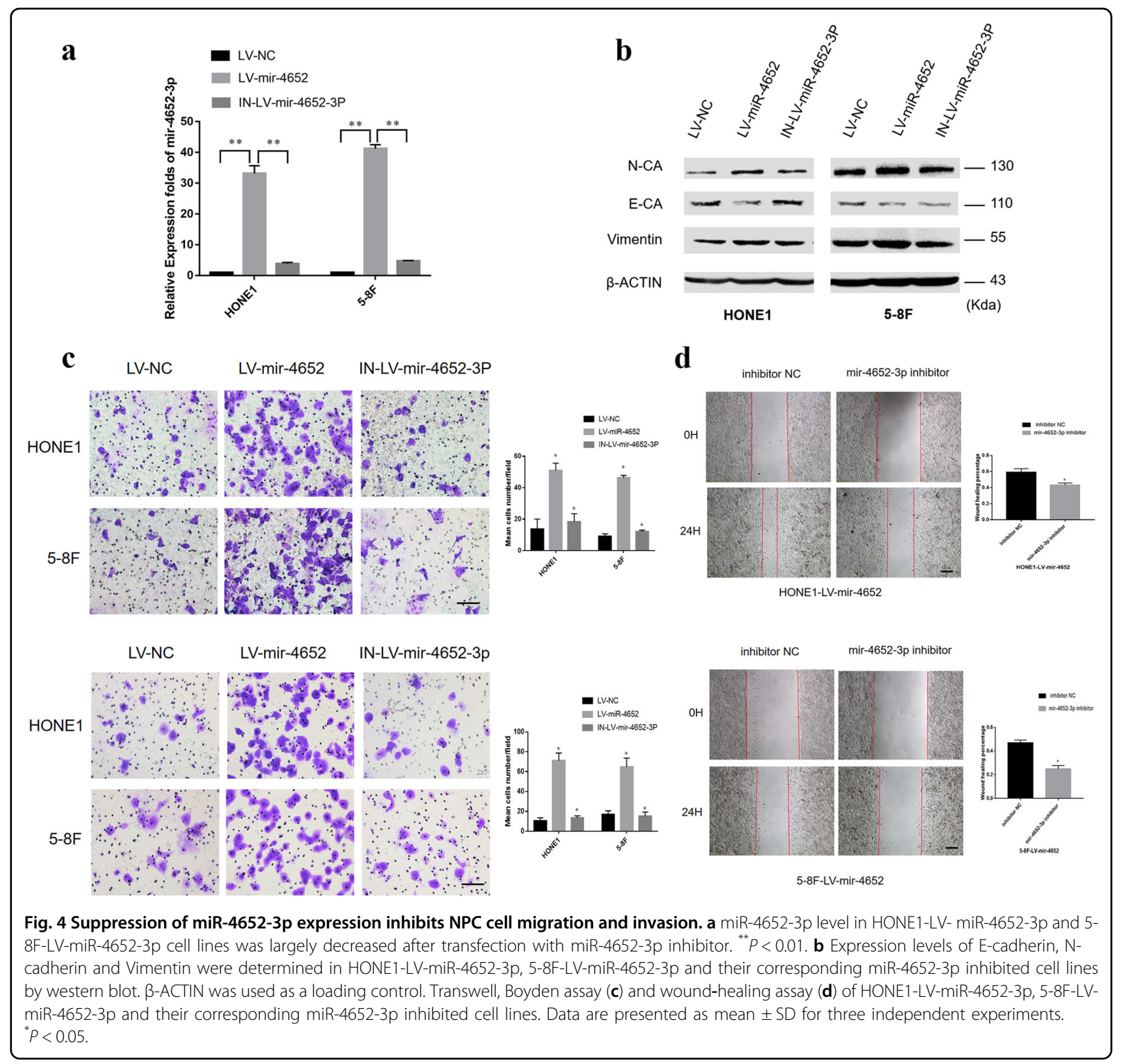

informed consent were obtained from patients. Two independent pathologists (Dr. Zhen Liu and Qingping Jiang) performed the blinded scoring. IHC staining scores were judged by the following criteria: A staining index (ranging from 0 to 12) was determined by the intensity of SPEN staining $(0=$ negative, $1=$ weakly positive, $2=$ moderate positive, $3=$ strongly positive), multiplied by the proportion score of immunopositive tumor cells $(0 \%=0$, $<10 \%=1,10 \%$ to $<50 \%=2,50 \%$ to $<75 \%=3, \geq 75 \%=4$ ). A minimum of 300 epithelial cells were counted for each sample and 3 or higher scores were classified as high SPEN expression.

\section{RNA interference}

The siRNAs specifically against SPEN and HIPK2 gene and their corresponding scrambled siRNAs were transfected into NPC cells in six-well plates using Lipofectamine 3000 transfection reagent (Invitrogen) according to the manufacturer's instructions. The scramble smallinterfering RNA (NC) and the siRNAs targeting SPEN (\#2, 5'-CCAAGATCGTACATATTAT-3'; \#3, 5'-GGA TCATGGTGCATCCACA-3'), and HIPK2 (\#1, $5^{\prime}$-GC UCACGGAAGCCAUUAUATT-3', \#2, 5'-GCGGACC ACACAACCUAAUTT-3') were synthesized and purchased from Ribobio (Guangzhou, China). 
$\mathbf{a}$

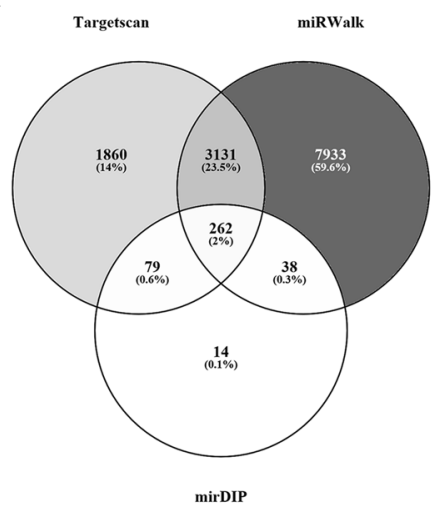

c

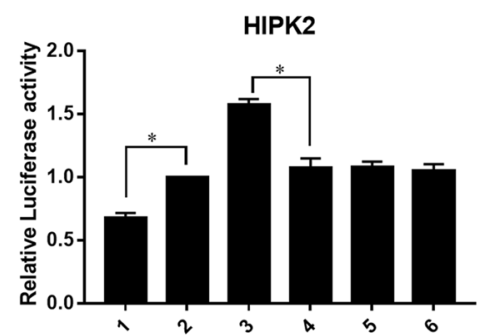

wt 3'UTR

PsiCHECK-2

$\mathrm{mt}$ 3'UTR

miR-4652-3P mimics

miR-4652-3P inhibitor

$$
\begin{array}{rrrrrr}
+ & - & + & - & - & - \\
- & + & - & + & - & - \\
- & - & - & - & + & + \\
+ & + & - & - & + & - \\
- & - & + & + & - & +
\end{array}
$$

b

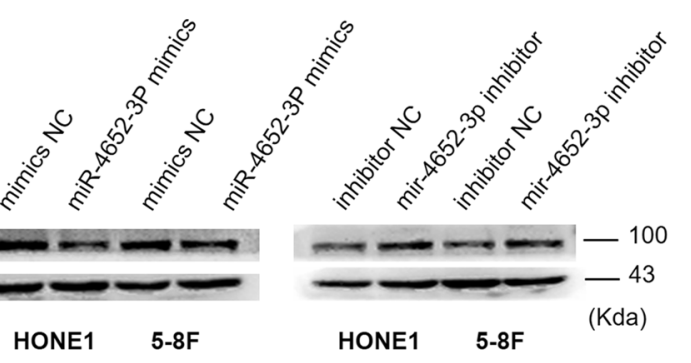

d

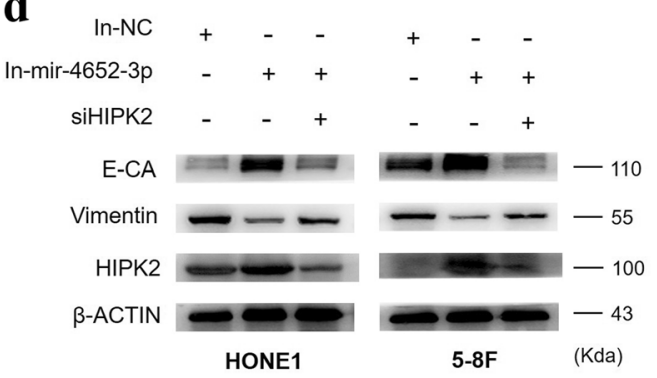

e

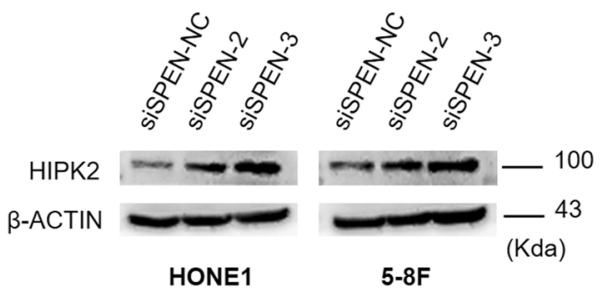

Fig. 5 miR-4652-3p directly targets HIPK2. a Targetscan, miRWalk, and mirDIP were used to predict target genes of miR-4652-3p. b HIPK2 expression was examined in miR-4652-3p-overexpressing or miR-4652-3p-inhibited cells by western blot, $\beta$-actin was used as a loading control. c Luciferase reporter assay was used to determine miR-4652-3p directly targets the $3^{\prime} U T R$ of HIPK2. ${ }^{*} P<0.05$. $\mathbf{d}$ Changes of E-cadherin, Vimentin and HIPK2 expression were detected by western blot in HONE1 and 5-8F cell lines after transfection of miR-4652-3p inhibitor or HIPK2 siRNAs. e Western blot of HIPK2 protein expression in HONE1 and 5-8F cells treated with SPEN siRNAs or corresponding control. $\beta$-actin served as a loading control.

In vitro cell migration, invasion and wound-healing assays

For migration assay, $1 \times 10^{4}$ cells in serum-free culture medium were added to the upper chamber, and the lower chamber was filled with $10 \%$ FBS culture media. Micro pore size of transwell membrane is $8 \mu \mathrm{m}$. After incubation, the filter was fixed with methanol, stained with crystal violet solution, and counted under a microscope in three random fields $(\times 200)$.

For invasion assay, transwell membranes were precoated with $35 \mu \mathrm{l}$ diluted matrix matrigel (BD Biosciences, USA) for $30 \mathrm{~min} .1 \times 10^{5}$ Cells adhering to the lower surface were counted the same way with the cell migration assay. Cells that migrated and invaded were quantified by counting cells in three random fields per filter.

For wound-healing assay, $4 \times 10^{6}$ cells were seed to confluence in a six-well plate, then cultured with serumfree medium. Artificial wound tracks were created by straight scraping confluent cell with a pipette tip. The ability of the cells to migrate into the wound area was assessed at 0 and $24 \mathrm{~h}$ after scratching. miRNA array following siSPEN Interference of SPEN in HONE1 cells was sent to Shanghai OE Biotech. Co., Ltd. (Shanghai, China) for miRNA biochip correlation analysis. The biochip used in this study was the SurePrint Agilent Human miRNA Microarrays (Release 21.0, ID:070156) obtained from (Agilent Technologies, Santa Clara, CA, USA).

\section{Establishment of NPC cell lines stably overexpressing miR- 4652-3p}

HONE1 and 5-8F cells were infected with a lentiviral expression vector carrying miR-4652-3p, which was constructed by GeneChem Co., Ltd. (Shanghai, China). MiR4652-3p overexpressing cells with green fluorescent protein signals were selected for further experiments by screening with puromycin. Total RNA was extracted from these cells and reverse transcribed into cDNA to measure the infection efficiency by qRT-PCR analysis. 


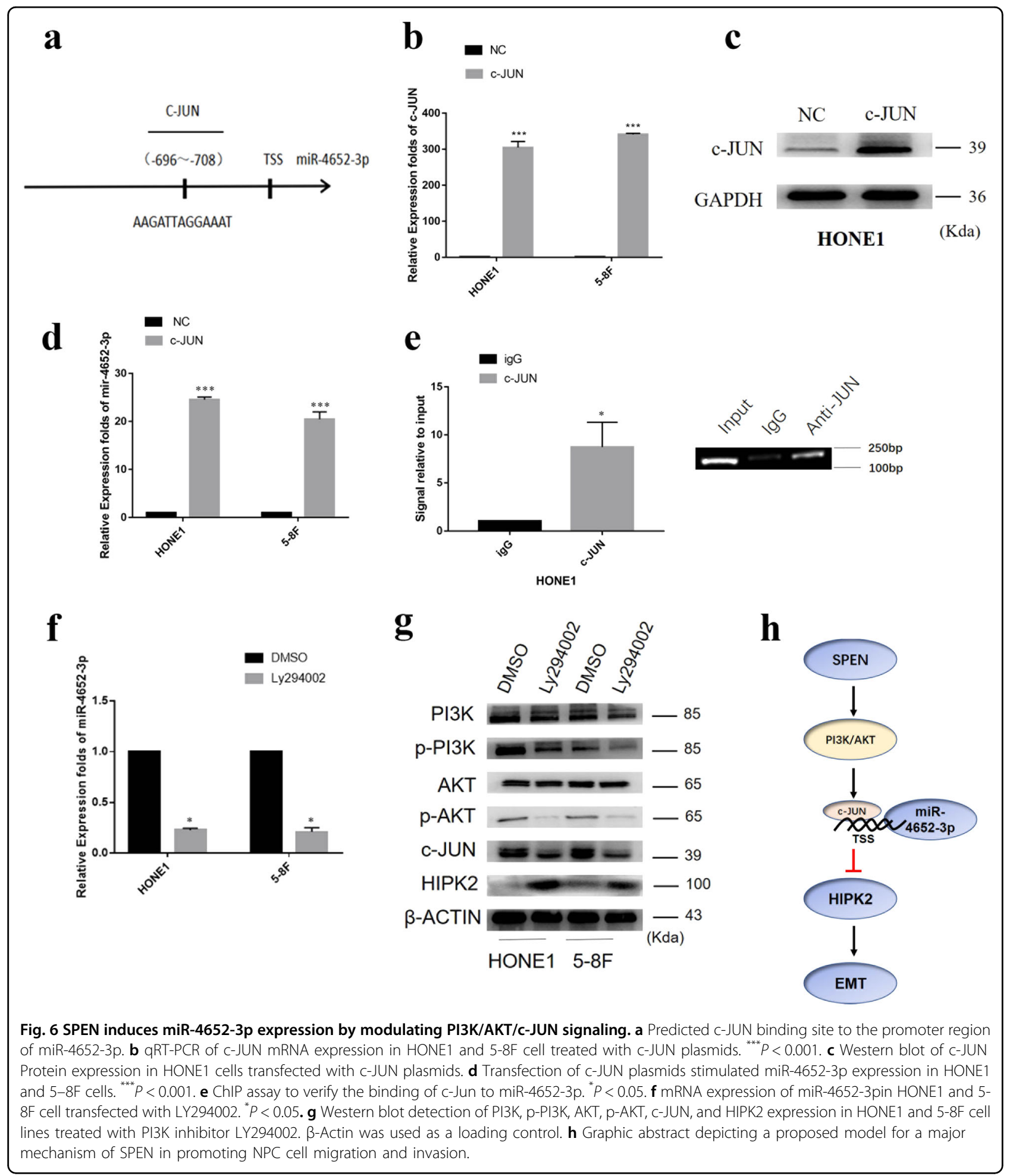

In vivo metastasis assay in nude mice

Five female BALB/c nude mice (4-week old) were randomly divided into four groups and injected with HONE1/58F-miR-4652-3P or HONE1/5-8F-miR-control cells. Briefly, $1 \times 10^{6}$ cells were injected intravenously through the tail vein of each nude mouse in a laminar flow cabinet. Four weeks after injection, the mice were sacrificed and examined for routine tissue processing. Lung tissues were then fluorescently imaged using the LT-9MACIMSYSPLUS whole-body imaging system (Light Tools Research, Encinitas, CA, USA). 
Subsequently, the Image J software (NIH, Bethesda, MD, USA) was used to quantifythe fluorescence signal intensity emitted from lung tissues. All animal experiments were conducted according to the standard institutional guidelines of Guangzhou Medical University. All animal experimental procedures were performed according to the Guidelines for the Care and Use of Laboratory Animals (NIH publications Nos. 80-23, revised 1996).

\section{Dual luciferase reporter assay}

The prediction software used predicted that HIPK2 is probably a direct target of miR-4652-3p. The $3^{\prime} \mathrm{UTR}$ fragment of HIPK2 was amplified using PCR primers and cloned into the psiCHECK- 2 vector. Site-directed mutagenesis of the miR-4652-3p binding site at the HIPK2 $3^{\prime}$ UTR was performed using the GeneTailor system (Invitrogen). The wild (wt) or mutant (mt) type of the HIPK2 3'UTR vector, psiCHECK-2 were co-transfected into the cells with miR-4652-3p mimics or inhibitors. The tests were independently performed in triplicate using a dual luciferase assay kit (Promega Corp., Madison, WI, USA) according tothe manufacturer's instructions.

\section{Chromatin immunoprecipitation (ChIP) assay}

ChIP analysis was performed using a ChIP assay kit (catalog: 17-371; Millipore, Billerica, MA, USA) according to the manufacturer's instructions to determine whether c-JUN binds to the promoter of miR-4652-3p. First, cells were transfected with a c-JUN plasmid and fixed with $1 \%$ formaldehyde. The cross-linked DNA is sonicated, cut to a length of 200-1000 base pairs, and then subjected to an immunoselection process, which requires the use of an anti-c-JUN antibody (1:50; Cell Signaling Technology (CST), Inc., Danvers, MA, USA). Ultimately, the agarose gel electrophoresis results reveal whether the DNA fragment of the putative cJUN binding site was present in the miR-4652-3p promoter.

\section{Statistical analysis}

Statistical analyses were performed using the SPSS 16.0 software (IBM Corp., Armonk, NY, USA) and GraphPad Prism v5.0 software (GraphPad Software Inc., La Jolla, CA, USA). Data are presented as the mean \pm SEM. For migration and invasion assays, statistical significance was determined using the Student's two-tailed $t$ test for two groups and one-way analysis of variance (ANOVA) for multiple groups. Immunohistochemistry analysis results were analyzed by the chi-square $\left(\chi^{2}\right)$ test. Survival analysis was performed using the Kaplan-Meier method. A $P$ value of $<0.05$ was considered as statistically significant. ${ }^{*} P<0.05,{ }^{* *} P<0.01$, and ${ }^{* * *} P<0.001$.

\section{Discussion}

SPEN has been reported to play a dual role in tumor development $t^{8-10}$. However, its biological function and molecular mechanism in NPC have not been elucidated. In this study, we first observed the upregulated mRNA expression of SPEN in NPC cells compared with immortalized nasopharyngeal epithelial (NP) cell lines (NP69 and SXSW-1489). In addition, IHC analysis revealed elevated SPEN protein expression level in NPC tissues compared with NP tissues. High expression of SPEN was identified as an independent predictor and cause of the poor outcome of NPC patients. Further, knocking down SPEN suppressed the migration and invasion of NPC cells. These findings was similar to Légaré and Liu et al.'s reports in breast cancer and ovarian cancer ${ }^{9,10}$, which demonstrated that dysregulation of SPEN was significantly involved in the development of NPC, suggesting that it plays a potential tumor metastasis promoter in NPC.

Molecular mechanisms underlying the migration and invasion of tumor cells have been intensively studied ${ }^{17,18}$. $\mathrm{PI} 3 \mathrm{~K} / \mathrm{AKT}$ is a key oncogenic signal that had been widely documented to activate EMT pathway and thus promote tumor migration, invasion, and metastasis ${ }^{19,20}$. In previous investigation, we also observed that dysregulated PI3K/AKT and its downstream EMT participated in some tumor-related gene-mediated NPC migration, invasion, and metastasis ${ }^{6,12}$. In this study, analog to Liu et al.'s data $^{10}$, we found that suppressing SPEN reduced PI3K/ $\mathrm{AKT}$ and its downstream EMT signal. Furthermore, cJun, an oncogenic transcription factor was also decreased in SPEN-suppressed NPC cells. These data further supported SPEN as a tumor metastasis promoter in NPC.

MiRNAs as intermediate regulators have been widely shown to participate in tumor-related gene-mediated signal network $^{21-23}$. In previous studies, we also observed that miR133a-3p, miR-5188, miR-3188, miR-374a, and miR-296-3p, respectively were modulated by VPS33B, $\mathrm{HBx}$, FOXO1, PDCD4, and HDGF involving in the tumor pathogenesis induced by these genes ${ }^{5,6,12,13,20,24,25}$. In order to further explore the molecular mechanism of metastasis induced by SPEN in NPC, Affymetrix miRNA array and qRT-PCR were used to identify the differential miRNAs in SPEN-knocking down cells. The data revealed that miR-4652-3p was a significantly positive-regulator of SPEN and might function as a tumor-promoted role in NPC cells. Consistent with our speculation, miR-4652-3p was found as a tumor metastasis promoter accelerating the metastasis of NPC. However, this data were not consistent with Li's report for miR-4652-3p in malignant meningioma ${ }^{26}$, which suggests the complexity of miR-4652-3p in tumors.

In order to explore the mechanisms by which miR4652-3p enhanced NPC cell migration, invasion and metastasis, bioinformatics were used to predict target genes through Targetscan, miRWalk, and mirDIP. Two hundred and sixty-two potential targets were found in all the above three databases, including HIPK2. The serine/ threonine kinase HIPK2, which was identified as a 
potential tumor suppressor in human neoplasms, is involved in transcriptional regulation and apoptosis ${ }^{27-32}$. Furthermore, HIPK2 inhibition was reported to promote EMT and subsequent cell invasion in bladder cancer ${ }^{27}$. In this study, we found that miR-4652-3p directly targeted HIPK2 and HIPK2 silencing abrogated the EMT signaling alteration caused by miR-4652-3p interference. These data demonstrated that miR-4652-3p targets HIPK2 to activate EMT signal and function as a promoter of tumor metastasis.

In prior study, we have shown that SPEN induces mir465-3p expression, However, the mechanism by which SPEN regulates miR-4652-3p expression has not been elucidated. It is well known that transcript factormediated miRNA transcription expression has been widely documented in tumors ${ }^{11,20,25}$. In this study, we first predicted the possible transcription factors binding to the miR-4652-3p promoter. Notably, among them, c-JUN was predicted as a potential transcription factor of miR-46523p. Overexpressed c-JUN markedly increased the expression of miR-4652-3p. In addition, the ChIP analysis indicated that c-JUN binds to the miR-4652-3p promoter.

$\mathrm{C}-\mathrm{JUN}$ is an oncogenic transcription factor known as a key regulator of major biological processes ${ }^{33}$ and recognized as a downstream positive regulator of PI3K/AKT signaling ${ }^{15,34-36}$. In previous studies, we also observed that c-JUN was induced by PI3K/AKT signal to modulate the expression of miRNAs expression in tumors including $\mathrm{NPC}^{14,24,37}$. Interestingly, we had observed that SPEN positively regulated the expression of PI3K/AKT/c-JUN and thus speculated that SPEN modulates the miR-4652-3p/HIPK2-mediated EMT signaling through the PI3K/AKT/c-JUN pathway. In line with this notion, suppressing $\mathrm{p}$-PI3K with its specific inhibitor LY294002 reduced the activation of $\mathrm{p}-\mathrm{AKT} / \mathrm{c}-\mathrm{JUN}$, which further downregulated miR-4652-3p expression, thereby increasing the HIPK2-induced inhibition of EMT signaling. Finally, we confirmed that SPEN was negatively correlated with HIPK2 protein expression in NPC cells and immortalized nasopharyngeal NP69 cells.

Overall, our study demonstrated for the first time that elevated SPEN might be used as a useful prognostic biomarker in NPC. Specifically, it activates PI3K/AKT/c-JUN to modulate miR-4652-3P/HIPK2 axis, which in turn activates EMT signaling and promotes NPC metastasis. Our findings reveal a novel pathway involved in NPC pathogenesis and broaden our understanding of NPC metastasis, which offer treatment options for improving the survival of patients with NPC.

\section{Acknowledgements}

This study was funded by the Natural Science Foundation of China (No. 81872198), Supporting Plan for Special Talents in Guangdong Province (No. 2016TQ03R466), Nature Science Fund of Guangdong Province (Nos.

2019A1515012194, 2017A030313702, 2017A030313701), Guangzhou Science and Technology Funding Projects (Nos. 201804010023, 201707010425), and the National Funds of Developing Local Colleges and Universities (No. B16056001). Shenzhen Key Laboratory of Viral Oncology (ZDSYS201707311140430), Seedling Program of Shenzhen Hospital of Southern Medical University (2017MM03).

\section{Author details}

'Affiliated Cancer Hospital \& Institute of Guangzhou Medical University, Guangzhou Municipal and Guangdong Provincial Key Laboratory of Protein Modification and Degradation, State Key Laboratory of Respiratory Disease, School of Basic Medical Sciences, Guangzhou Medical University, Guangzhou 510095 Guangdong, China. ${ }^{2}$ Department of Pediatric Otorhinolaryngology, Shenzhen Key Laboratory of Viral Oncology, The Clinical Innovation \& Research Centre, Shenzhen Hospital, Southern Medical University, Shenzhen,

Guangdong, China. ${ }^{3}$ Department of Pathology, The Sixth Affiliated Hospital of Sun Yat-sen University, Guangzhou, Guangdong, China. ${ }^{4}$ Department of Oncology, The Fifth Affiliated Hospital of Guangzhou Medical University, Guangzhou, Guangdong, China. ${ }^{5}$ Department of Pathology, Third Affiliated Hospital of Guangzhou Medical University, Guangzhou, Guangdong, China. ${ }^{6}$ Department of Breast Surgery, Guiyang Maternal and Child Healthcare Hospital, Guiyang 550003 Guizhou, China. 'E.N.T. Department of Nanfang Hospital, Southern Medical University, Guangzhou, Guangdong, China

\section{Author contributions}

Zhen Liu, Chao Cheng and Xiong Liu designed the study. Yumin Lv, Liu Yang, Jingjing He, Xingyu Tao, Yingying Hu, Yuting Ma, Yun Su, Liyang Wu and Yang Li conducted the experiments. Zhen Liu and Yang Li wrote the manuscript. Guifang Yu, Yan Huang, Shu Liu and Qingping Jiang formed the data analysis and helped to draft the manuscript. All authors read and approved the final manuscript.

\section{Data availability}

The data generated, used, and analyzed in the current study are available from the corresponding author in response to reasonable request.

\section{Conflict of interest}

The authors declare that they have no conflict of interest.

\section{Publisher's note}

Springer Nature remains neutral with regard to jurisdictional claims in published maps and institutional affiliations.

Received: 12 December 2019 Revised: 10 June 2020 Accepted: 15 June 2020

Published online: 02 July 2020

\section{References}

1. Wei, K. R. et al. Nasopharyngeal carcinoma incidence and mortality in China, 2013. Chin. J. Cancer 36, 90 (2017).

2. Tang, L. L. et al. Global trends in incidence and mortality of nasopharyngeal carcinoma. Cancer Lett. 374, 22-30 (2016).

3. Lee, A. W. et al. The battle against nasophanyngeal cancer. Radiother. Oncol. 104, 272-278 (2012).

4. Cai, L. M. et al. EBV-miR-BART7-3p promotes the EMT and metastasis of nasopharyngeal carcinoma cells by suppressing the tumor suppressor PTEN. Oncogene 34, 2156-2166 (2015).

5. Deng, X. et al. miR-296-3p negatively regulated by nicotine stimulates cytoplasmic translocation of c-Myc via MK2 to suppress chemotherapy resistance. Mol. Ther.: J. Am. Soc. Gene Ther. 26, 1066-1081 (2018).

6. Zhen, Y. et al. miR-374a-CCND1-pPI3K/AKT-c-JUN feedback loop modulated by PDCD4 suppresses cell growth, metastasis, and sensitizes nasopharyngeal carcinoma to cisplatin. Oncogene 36, 275-285 (2017).

7. Sanchez-Pulido, L. et al. SPOC: a widely distributed domain associated with cancer, apoptosis and transcription. BMC Bioinform. 5, 91 (2004).

8. Legare, S., Chabot, C. \& Basik, M. S.P.E.N. a new player in primary cilia formation and cell migration in breast cancer. Breast Cancer Res. 19, 104 (2017). 
9. Feng, Y. et al. Drosophila split ends homologue SHARP functions as a positive regulator of Wnt/beta-catenin/T-cell factor signaling in neoplastic transformation. Cancer Res. 67, 482-491 (2007).

10. Liu, D., Yang, Y., Yan, A. \& Yang, Y. SPOCD1 accelerates ovarian cancer progression and inhibits cell apoptosis via the PI3K/AKT pathway. OncoTargets Ther. 13, 351-359 (2020)

11. Li, Y. et al. Chemical compound cinobufotalin potently induces FOXO1stimulated cisplatin sensitivity by antagonizing its binding partner MYH9. Signal Transduct. Target. Ther. 4, 48 (2019).

12. Liang, Z. et al. VPS33B interacts with NESG1 to modulate EGFR/PI3K/AKT/CMyc/P53/miR-133a-3p signaling and induce 5-fluorouracil sensitivity in nasopharyngeal carcinoma. Cell Death Dis. 10, 305 (2019).

13. Zhao, M. et al. Dual roles of miR-374a by modulated c-Jun respectively targets CCND1-inducing PI3K/AKT signal and PTEN-suppressing Wnt/ beta-catenin signaling in non-small-cell lung cancer. Cell Death Dis. 9 78 (2018).

14. Lin, X. et al. RNA-binding protein LIN28B inhibits apoptosis through regulation of the AKT2/FOXO3A/BIM axis in ovarian cancer cells. Signal Transduct. Target. Ther. 3, 23 (2018).

15. Ye, Y. et al. EBV-miR-BART1 is involved in regulating metabolism-associated genes in nasopharyngeal carcinoma. Biochem. Biophys. Res. Commun. 436, 19-24 (2013).

16. Chen, $X$. et al. SP1-induced IncRNA-ZFAS1 contributes to colorectal cancer progression via the miR-150-5p/NEGFA axis. Cell Death Dis. 9, 982 (2018).

17. Cheng, W. C. et al. RAB27B-activated secretion of stem-like tumor exosomes delivers the biomarker microRNA-146a-5p, which promotes tumorigenesis and associates with an immunosuppressive tumor microenvironment in colorectal cancer. Int. J. Cancer 145, 2209-2224 (2019).

18. Ge, Q. et al. miR-4324-RACGAP1-STAT3-ESR1 feedback loop inhibits proliferation and metastasis of bladder cancer. Int. J. Cancer 144, 3043-3055 (2019).

19. Manoel-Caetano, F. S. et al. Upregulation of the APE1 and H2AX genes and miRNAs involved in DNA damage response and repair in gastric cancer. Genes Dis. 6, 176-184 (2019).

20. Lin, X. et al. HBX-induced miR-5188 impairs FOXO1 to stimulate beta-catenin nuclear translocation and promotes tumor stemness in hepatocellular carcinoma. Theranostics 9, 7583-7598 (2019).

21. Qiu, Y. Y. et al. LncRNA-MEG3 functions as a competing endogenous RNA to regulate Treg/Th17 balance in patients with asthma by targeting microRNA17/ RORgammat. Biomed. Pharmacother. 111, 386-394 (2019).

22. Zhang, H. et al. Exosomal circRNA derived from gastric tumor promotes white adipose browning by targeting the miR-133/PRDM16 pathway. Int. J. Cancer 144, 2501-2515 (2019).
23. Thakur, $C$. et al. Loss of mdig expression enhances DNA and histone methylation and metastasis of aggressive breast cancer. Signal Transduct. Target. Ther. 3, 25 (2018).

24. Zhao, M. et al. miR-3188 regulates nasopharyngeal carcinoma proliferation and chemosensitivity through a FOXO1-modulated positive feedback loop with mTOR-p-PI3K/AKT-c-JUN. Nat. Commun. 7, 11309 (2016).

25. Fu, Q. et al. miRomics and proteomics reveal a miR-296-3p/PRKCA/FAK/Ras/cMyc feedback loop modulated by HDGF/DDX5/beta-catenin complex in lung adenocarcinoma. Clin. Cancer Res. 23, 6336-6350 (2017).

26. Li, T. et al. LINC00702/miR-4652-3p/ZEB1 axis promotes the progression of malignant meningioma through activating Wnt/beta-catenin pathway. Biomed. Pharmacother. 113, 108718 (2019).

27. Tan, M. et al. Downregulation of homeodomain-interacting protein kinase-2 contributes to bladder cancer metastasis by regulating Wnt signaling. J. Cell. Biochem. 115, 1762-1767 (2014).

28. Hofmann, T. G., Glas, C. \& Bitomsky, N. HIPK2: a tumour suppressor that controls DNA damage-induced cell fate and cytokinesis. BioEssays: N. Rev. Mol., Cell. Dev. Biol. 35, 55-64 (2013).

29. D'Orazi, G. et al. Homeodomain-interacting protein kinase-2 phosphorylates p53 at Ser 46 and mediates apoptosis. Nat. Cell Biol. 4, 11-19 (2002).

30. Kim, E. A. et al. Homeodomain-interacting protein kinase 2 (HIPK2) targets beta-catenin for phosphorylation and proteasomal degradation. Biochem. Biophys. Res. Commun. 394, 966-971 (2010).

31. Pierantoni, G. M. et al. The homeodomain-interacting protein kinase 2 gene is expressed late in embryogenesis and preferentially in retina, muscle, and neural tissues. Biochem. Biophys. Res. Commun. 290, 942-947 (2002).

32. Nardinocchi, L. et al. Transcriptional regulation of hypoxia-inducible factor 1alpha by HIPK2 suggests a novel mechanism to restrain tumor growth. Biochim. Biophys. Acta 1793, 368-377 (2009).

33. Lin, $\mathrm{X}$. et al. Silencing MYH9 blocks HBx-induced GSK3beta ubiquitination and degradation to inhibit tumor stemness in hepatocellular carcinoma. Signal Transduct. Target. Ther. 5, 13 (2020).

34. Zeng, Q. et al. Inhibition of ZIP4 reverses epithelial-to-mesenchymal transition and enhances the radiosensitivity in human nasopharyngeal carcinoma cells. Cell Death Dis. 10, 588 (2019).

35. Liu, W. et al. DCLK1 promotes epithelial-mesenchymal transition via the PI3K Akt/NF-kappaB pathway in colorectal cancer. Int. J. Cancer 142, 2068-2079 (2018).

36. Nepstad, I. et al. Effects of insulin and pathway inhibitors on the PI3K-AktmTOR phosphorylation profile in acute myeloid leukemia cells. Signal Transduct. Target. Ther. 4, 20 (2019).

37. $\mathrm{Yu}, \mathrm{X}$. et al. Loss of connective tissue growth factor as an unfavorable prognosis factor activates miR-18b by PI3K/AKT/C-Jun and C-Myc and promotes cell growth in nasopharyngeal carcinoma. Cell Death Dis. 4, e634 (2013). 